\title{
Organization of the Human Inferior Parietal Lobule Based on Receptor Architectonics
}

\author{
Svenja Caspers ${ }^{1}$, Axel Schleicher ${ }^{2}$, Mareike Bacha-Trams ${ }^{1,3}$, Nicola Palomero-Gallagher ${ }^{1}$, Katrin Amunts ${ }^{1,4,5}$ and Karl Zilles ${ }^{1,2,4}$ \\ ${ }^{1}$ Institute of Neuroscience and Medicine (INM-1, INM-2), Research Centre Jülich, 52425 Jülich, Germany, ${ }^{2} \mathrm{C}$. and O. Vogt Institute \\ for Brain Research, Heinrich-Heine-University Düsseldorf, 40001 Düsseldorf, Germany, ${ }^{3}$ Max-Planck-Institute for Human Cognitive \\ and Brain Sciences, 04103 Leipzig, Germany, ${ }^{4}$ JARA-BRAIN, Jülich-Aachen Research Alliance, 52425 Jülich, Germany and \\ ${ }^{5}$ Department of Psychiatry, Psychotherapy, and Psychosomatics, RWTH Aachen University, 52072 Aachen, Germany
}

Address correspondence to Dr Svenja Caspers, Institut für Neurowissenschaften und Medizin, INM-2, Forschungszentrum Jülich, 52425 Jülich, Germany. Email: s.caspers@fz-juelich.de.

Human inferior parietal lobule (IPL) plays a key role in various cognitive functions. Its functional diversity, including attention, language, and action processing, is reflected by its structural segregation into 7 cytoarchitectonically distinct areas, each with characteristic connectivity patterns. We hypothesized that commonalities of the cytoarchitectonic, connectional, and functional diversity of the IPL should be reflected by a correlated transmitter receptor-based organization. Since the function of a cortical area requires a well-tuned receptor balance, the densities of $\mathbf{1 5}$ different receptors were measured in each IPL area. A hierarchical cluster analysis of the receptor balance revealed a tripartite segregation of the IPL into a rostral, middle, and caudal group. Comparison with other cortical areas showed strong similarities with Broca's region for all 3 groups, with the superior parietal cortex for the middle, and with extrastriate visual areas for the caudal group. Notably, caudalmost area PGp has a receptor fingerprint very similar to that of ventral extrastriate visual cortex. We therefore propose a new organizational model of the human IPL, consisting of 3 clusters, which corresponds to its known cytoarchitectonic, connectional, and functional diversity at the molecular level. This might reflect a general organizational principle of human IPL, beyond specific functional domains.

Keywords: architecture, cerebral cortex, inferior parietal lobe, structural segregation, transmitter receptors

\section{Introduction}

The human inferior parietal lobule (IPL) comprises the supramarginal gyrus rostrally and the angular gyrus caudally. Brodmann (1909) subdivided the human IPL into 2 cytoarchitectonical areas: BA 40 rostrally and BA 39 caudally. Electrophysiological studies in macaques and functional neuroimaging in humans suggest, however, a functionally much more heterogeneous IPL than Brodmann's map suggests. The cytoarchitectonic analysis of von Economo and Koskinas (1925) hinted at a more detailed parcellation. They defined several subtypes within the 2 main IPL areas (termed PF and PG) but could not establish them as unique.

In monkeys, rostral IPL is involved in sensorimotor integration and contains mirror neurons (Fogassi et al. 2005), whereas caudal IPL was found to participate in spatial attention, visuomotor, and auditory processes (Mountcastle et al. 1975; Hyvärinen 1982; Pandya and Seltzer 1982; Seltzer and Pandya 1984; Rozzi et al. 2008). A comparable functional segregation was found in humans: Rostral human IPL seems to be involved in motor planning and action-related functions and is part of the human mirror neuron system (Iacoboni 2005; Rizzolatti 2005; Keysers and Gazzola 2009; Caspers et al. 2010). The left caudal
IPL is active during language-related tasks with focus on semantic and phonological issues (Price 2000; Vigneau et al. 2006), while the right caudal IPL was found to be involved in spatial and nonspatial attention as well as motor preparation (Fink et al. 2001; Corbetta et al. 2008).

This functional segregation found a structural correlation in recent observations. In monkeys, 4 areas were identified on the lateral surface of the IPL and 2 areas on the caudal part of the parietal operculum within the Sylvian fissure (Pandya and Seltzer 1982; Gregoriou et al. 2006). In humans, a similar parcellation could be established. Seven cytoarchitectonically distinct areas were recently described, 5 of which are located on the lateral surface, whereas the remaining 2 areas are located on the caudal parietal operculum (Caspers et al. 2006, 2008) (Fig. 1).

The functional and architectonical diversity of the IPL are also reflected by differential connectivity patterns of the areas. The fiber tracts between the IPL and other cortical areas change from rostral to caudal, as demonstrated in a recent diffusion tensor imaging study (Caspers, Eickhoff, et al. 2011): Whereas rostral IPL areas show strong connections with inferior frontal, motor, premotor, and somatosensory areas, caudal IPL areas are more strongly connected with posterior parietal, higher visual, and temporal areas. Areas in the middle of the IPL are connected with the targets of both rostral and caudal IPL areas. A comparable differential connectivity pattern was found by means of connectivity-based parcellation of the IPL (Mars et al. 2011). This pattern strikingly resembles that found in tracer studies in macaques (Cavada and GoldmanRakic 1989a, 1989b; Andersen et al. 1990; Rozzi et al. 2006). Thus, the structural, functional, and connectivity data favor the concept of a highly segregated brain region.

Mapping the regional and laminar distribution patterns of different receptors in the cerebral cortex proved to be a powerful tool for detecting functionally meaningful cortical parcellations (Zilles and Palomero-Gallagher 2001; Zilles, Palomero-Gallagher, et al. 2002; Zilles, Schleicher, et al. 2002; Zilles and Amunts 2009). Not only primary motor, premotor, and primary somatosensory cortices (Geyer et al. 1997, 1998) but also higher order areas such as Broca's region (Amunts et al. 2010), the striate and extrastriate visual cortex (Eickhoff et al. 2007,2008 ) as well as the superior parietal lobule (Scheperjans, Grefkes, et al. 2005; Scheperjans, Palomero-Gallagher, et al. 2005), the cingulate cortex (Palomero-Gallagher et al. 2009), and the superior temporal gyrus (Morosan et al. 2005) have been subdivided into distinct receptor-architectonical entities. Moreover, it has been demonstrated that cortical areas with similar receptor expression patterns are nodes in the same functionally distinct neural network (Zilles, Palomero-Gallagher, et al. 2002; Zilles and Amunts 2009). 


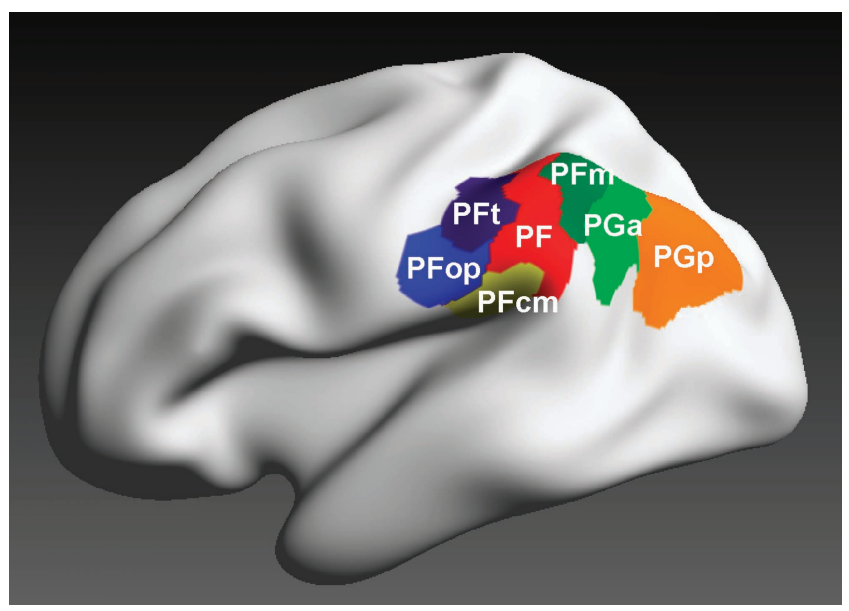

Figure 1. 3D reconstructed maximum probability maps of the 7 cytoarchitectonically defined IPL areas PFt, PFop, PF, PFm, PFcm, PGa, and PGp (Caspers et al. 2006, 2008) on the inflated lateral surface view of the Montreal Neurological Institute single subject template.

We therefore used quantitative in vitro autoradiography for multireceptor mapping in the human IPL to understand the molecular basis underlying its structural and functional heterogeneity. We studied the densities of multiple receptor binding sites and the regionally specific balances between them in each of the 7 cytoarchitectonic areas of the IPL (Caspers et al. 2006, 2008). Their regional receptor distribution patterns were then compared with those of cortical areas outside the IPL to gain further insight into the receptor-based organization of the cerebral cortex, similarities and dissimilarities of receptor expression patterns between distinct functional systems, and the putative relationships of the different IPL areas with various functional systems.

\section{Material and Methods}

\section{Postmortem Tissue Extraction and Preparation}

Nine human postmortem hemispheres (6 right and 3 left) were obtained from body donors without any known history of neurological or psychiatric disorders, according to legal requirements. Brains were removed from the skull within $24 \mathrm{~h}$ post-mortem (Table 1 ).

Each hemisphere was cut into 5 or 6 coronal slabs of about $25-30 \mathrm{~mm}$ thickness each. After shock freezing of the tissue at $-50{ }^{\circ} \mathrm{C}$ for $10 \mathrm{~min}$ in liquid isopentane to avoid freezing artifacts within the cortex, the slabs were stored at $-70{ }^{\circ} \mathrm{C}$. Subsequently, each slab was cut into serial coronal sections $(20 \mu \mathrm{m}$ thickness $)$ at $-20^{\circ} \mathrm{C}$, using a large-scale cryostat microtome. The sections were thaw mounted onto glass slides prior to further processing (Fig. $2 A, B$ ).

\section{Autoradiographic Labeling of Receptors}

For quantitative autoradiographic multireceptor analysis of the human IPL, alternating sections were 1) incubated with a tritiated receptor ligand, 2) incubated with a tritiated ligand and a nonradioactive displacing compound to measure the nonspecific binding of the receptor, or 3) stained for cell bodies using a modified silver stain (Merker 1983). Thus, a group of sections at the same sectioning level provided information about the receptor distribution of different receptors as well as the corresponding cytoarchitecture.

In total, distribution of 15 different receptors from 6 classical neurotransmitter systems was investigated in the present study: glutamatergic ( $\alpha$-amino-3-hydroxy-5-methyl-4-isoxazolepropionic acid [AMPA], kainate, $N$-methyl-D-aspartate [NMDA]), $\gamma$-aminobutyric acid (GABA)ergic $\left(\mathrm{GABA}_{\mathrm{A}^{-}}, \mathrm{GABA}_{\mathrm{B}^{-}}, \mathrm{GABA}_{\mathrm{A}^{-}}\right.$-associated benzodiazepine-binding sites), cholinergic (nicotinic, muscarinic $\left.M_{1}, M_{2}, M_{3}\right)$, adrenergic $\left(\alpha_{1}, \alpha_{2}\right)$,

\begin{tabular}{|c|c|c|c|c|c|}
\hline \multicolumn{6}{|c|}{$\begin{array}{l}\text { Table } 1 \\
\text { Data of postmortem bra }\end{array}$} \\
\hline $\begin{array}{l}\text { Brain } \\
\text { no. }\end{array}$ & Hemisphere & Sex & $\begin{array}{l}\text { Age } \\
\text { (years) }\end{array}$ & Cause of death & $\begin{array}{l}\text { Postmortem } \\
\text { delay (h) }\end{array}$ \\
\hline 1 & Left & Female & 77 & Coronary heart disease & 10 \\
\hline 2 & Right & Male & 72 & Cardiac arrest & 8 \\
\hline 3 & Left/right & Female & 77 & Pulmonary edema & 18 \\
\hline 4 & Left/right & Male & 78 & Multiorganic failure & 12 \\
\hline 5 & Left/right & Female & 75 & Bronchial cancer & 16 \\
\hline 6 & Right & Male & 79 & $\begin{array}{l}\text { Sudden cardiac death, } \\
\text { chronic cardiac insufficiency }\end{array}$ & 12 \\
\hline
\end{tabular}

serotoninergic $\left(5-\mathrm{HT}_{1 \mathrm{~A}}, 5-\mathrm{HT}_{2}\right)$, and dopaminergic $\left(\mathrm{D}_{1}\right)$. Supplementary Table S1 provides an overview of the binding protocols for all receptors studied. For all receptors and cases, nonspecific binding was less than 5\% of the total binding. Thus, the total binding of each receptor could be accepted as an estimate of the specific binding.

After incubation with the tritiated ligands, the sections were coexposed with plastic scales of known concentrations of radioactivity to films sensitive to $\beta$-radiation (Hyperfilm, Amersham or Kodak BioMax MR films) for 8-18 weeks, depending on the receptor (Supplementary Table S1). The resulting autoradiographs (Fig. 2C) represent the regional and laminar distribution of receptor-binding sites. The gray value distribution within the autoradiographs was nonlinearly correlated with the local concentration of the radioactivity. The known radioactivity of the coexposed plastic standards was calibrated to brain homogenates with known protein concentration to allow transformation of gray values of the autoradiographs into total binding (femtomole per milligram protein), displayed within linearized images (Fig. 2D). For a more comfortable visualization of the local receptor distribution within the sections, the linearized images were contrast enhanced, smoothed, and pseudocolor coded in a spectral sequence (Fig. 2E). After digitization of the films, receptor concentrations were measured as described previously (Zilles, Palomero-Gallagher, et al. 2002; Zilles, Schleicher, et al. 2002; Zilles et al. 2004; Schleicher et al. 2009).

\section{Quantitative Receptor Analysis of the IPL}

For the analysis of receptor densities in different cortical areas, regions of interest (ROIs) were defined, using the cell body-stained sections adjacent to each group of autoradiographs. ROIs covered the whole cortical width. Seven IPL areas (PFt, PFop, PF, PFm, PFcm, PGa, and PGp) were identified based on cytoarchitectonic criteria as published (Caspers et al. 2006). To assure that the 7 cytoarchitectonically defined IPL areas were identified in a similar way by their receptor distribution pattern and for consecutive analysis of laminar receptor distributions, borders between the IPL areas and surrounding cortical regions were delineated within all receptor autoradiographs. For delineation of these borders, a multivariate statistical algorithm-based approach was used (Schleicher et al. 2005, 2009). This algorithm uses laminar information of the gray-level index distribution within each section. Feature vectors described the shape of each profile and thus reflected the underlying laminar receptor architecture. Using the Mahalanobis distance as distance measure, adjacent blocks of profiles could be compared by means of Hotelling's $T^{2}$-test for significant differences in Mahalanobis distance (Bonferroni-corrected for multiple comparisons). Delineation of areas was possible since profiles from different cortical areas differed considerably in shape, which results in larger distances between them (Zilles et al. 2004; Schleicher et al. 2009). This procedure was repeatedly carried out for blocks of profiles (ranging from 10 to 24 profiles per block) to improve signal-to-noise ratio (Figs 3 and 4).

After delineation of the IPL areas, respective ROIs were defined (3 per area per hemisphere) for subsequent averaging where receptor densities of the 15 different receptors were measured (Zilles, Palomero-Gallagher, et al. 2002; Zilles, Schleicher, et al. 2002; Schleicher et al. 2009), using MATLAB 7.7 (The MathWorks Inc.). For each of the 7 IPL areas, receptor density values were averaged over the 9 hemispheres, providing a mean value for each receptor in each area.

The receptor balance of each area was visualized as receptor fingerprint. The mean receptor densities (averaged over all cortical 

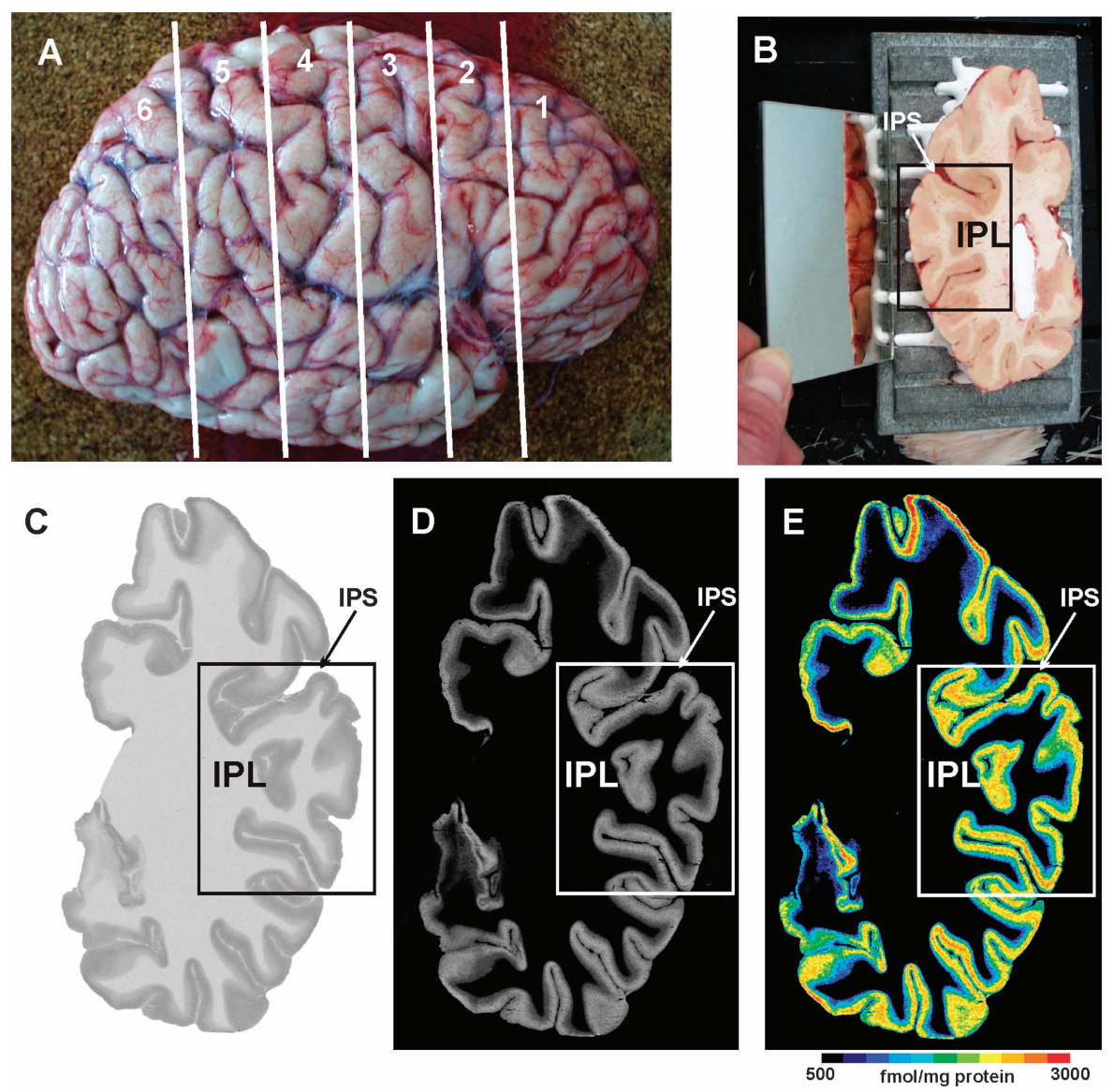

Figure 2. Quantitative in vitro receptor autoradiography. (A) Right human hemisphere prior to sectioning into 6 slabs (white lines) for further processing. (B) Blockface of a frozen slab on the cryotome with the labeled ROI in the present study (IPL). The mirror on the left side provides a lateral view of the tissue slab. $(C)$ Autoradiograph of the GABA $B$ receptor of the same slab, ROI marked by a box. (D) Scaled autoradiograph (same as in C) with gray values reflecting the receptor concentrations, calculated from coexposed plastic scales of known radioactivity concentrations. (E) Pseudocolor-coded autoradiograph (same as in C). The colors indicate receptor concentrations, from black for low to red for high concentrations (for concentrations in femtomole per milligram protein, see color bar). IPS: intraparietal sulcus.

layers) for each receptor type (averaged over hemispheres) were registered in a polar plot, which represent the characteristic receptor fingerprint of each area. These fingerprints could consecutively be compared with regard to their size and shape by using a unified scaling for each receptor for all areas (Zilles, Palomero-Gallagher, et al. 2002; Zilles, Schleicher, et al. 2002). This allows a direct comparison of different cortical areas to reveal similarities and differences in their receptor distribution pattern.

For comparison with other cortical areas, additional ROIs were defined based on published cytoarchitectonic and macroanatomical criteria. Cortical areas were chosen to optimally categorize the IPL areas in relation to other cortical areas. Therefore, ROIs within primary as well as higher order association cortices were defined: primary motor cortex (M1; Geyer et al. 1996); primary somatosensory areas 3b and 1 (S1_3b, S1_1; Geyer et al. 1999, 2000); primary and secondary visual cortex (V1, V2; Amunts et al. 2000); ventral extrastriate visual cortex lateral to V1 and V2 (mainly V3v, V4v; Rottschy et al. 2007); primary and secondary auditory cortex (A1, A2; Morosan et al. 2001); Broca's area (area 44; Amunts et al. 1999, 2010); and posterior superior parietal lobule (area 7A; Scheperjans, Hermann, et al. 2008; Scheperjans, Eickhoff, et al. 2008). ROIs within Broca's area and the superior parietal lobule were chosen representatively. It was shown that both these regions could be parcellated into several subdivisions based on their receptor architecture. But the architecture within these subdivisions was very similar to each other, especially as compared with other cortical areas (Scheperjans, Grefkes, et al. 2005; Scheperjans, PalomeroGallagher, et al. 2005; Amunts et al. 2010). Thus, including more subdivisions within the present analysis would not add substantial new information for a basic functional classification of the IPL areas.

\section{Statistical Analysis}

The mean density values of all 15 receptors studied were combined into a feature vector for each area. Since absolute receptor concentrations differed considerably between receptor types, all values were $z$-transformed across areas prior to any further analysis. The transformation enabled analyses where all receptors had equal weight. Similarities and differences between receptor distribution patterns of areas were analyzed by means of a hierarchical cluster analysis (MATLAB 7.7, Statistics Toolbox, The MathWorks Inc.), using Euclidean distances in combination with the Ward linkage method. Euclidean distances between feature vectors became smaller the more similar the areas were.

In addition, areal feature vectors were further analyzed by means of a multidimensional scaling (MDS; Systat 12) to detect similar and dissimilar groups of areas. MDS resulted in a 2D display of the 15 dimensional receptor feature vectors. To identify those receptors, which accounted most for separation into different clusters, a multivariate canonical discriminant analysis was performed (Systat 12).

All these analyses were carried out on the mean receptor densities of all IPL areas. The hierarchical cluster analysis was also conducted for the comparison of IPL with other cortical areas.

\section{Results}

\section{Receptor Mapping of IPL Areas}

The measurement of the receptor density of each area from the cortical surface to the cortex/white matter border demonstrates the quantitative laminar-specific distribution of the receptors. 

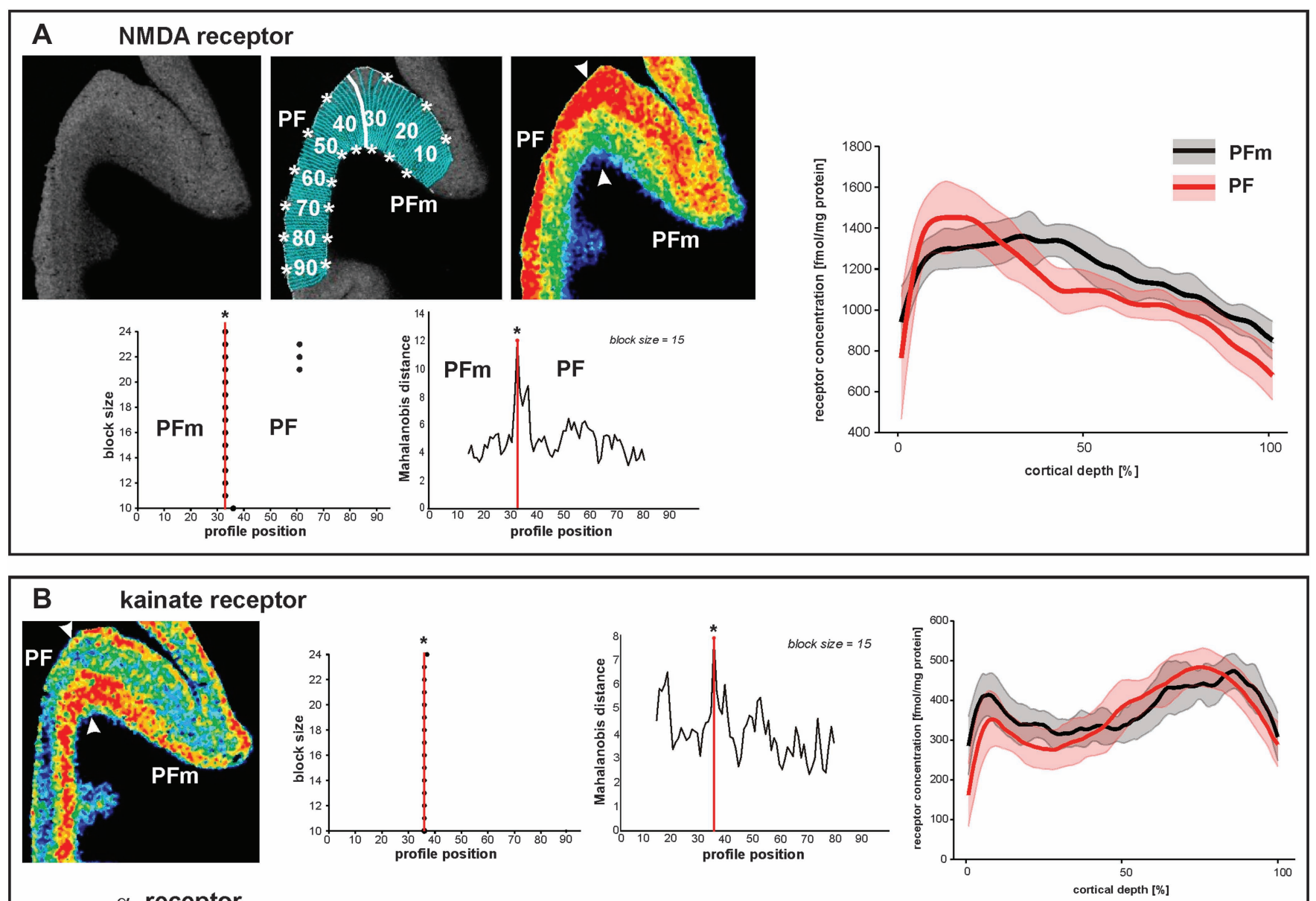

$\alpha_{2}$ receptor
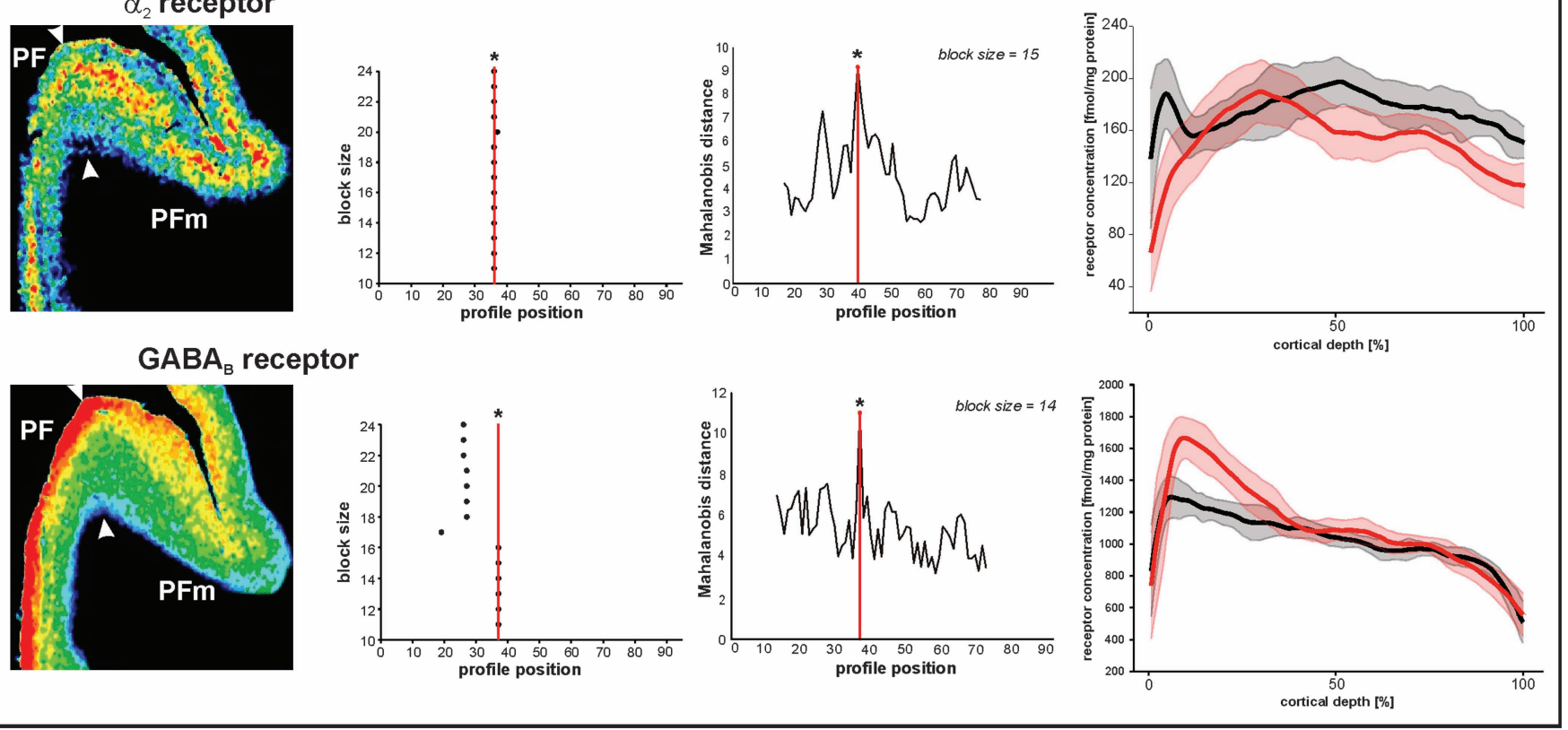

Figure 3. Parcellation of IPL based on receptor distribution patterns. (A) Part of a receptor autoradiograph (NMDA receptor) of the IPL (border region between areas PF and PFm as shown in Figure $5 A$ for whole IPL). The autoradiograph of the cortical ribbon (upper left) was covered by traverses running perpendicular to the cortical layers (upper middle) and pseudocolor coded for visualization purposes only (upper right). Results of the algorithmic parcellation are shown below: the left graph shows the significant maxima of varying block sizes (ranging from 10 to 24); it indicates a consistently occurring border between 2 cortical areas at profile location 33 . Right next to it, a line plot shows the Mahalanobis distances between neighboring blocks of profiles; it confirms the location of the maximal distance, and thus, the maximal dissimilarity between adjacent profiles at profile location 33, which defines an architectural border. The border is also labeled in the autoradiographs above. The graph on the right side of $(A)$ shows the laminar distribution (with standard deviations) of the NMDA receptor throughout the cortical width $(0 \%$ at the transition from the pial surface to layer I; $100 \%$ at the transition from layer $\mathrm{VI}$ to the white matter) in areas PF and PFm. The profiles differ between both areas. (B) Parcellation of the same part of the cortex by 3 other receptors (kainate, $\alpha_{2}$, and GABA $)$. Figures and graphs of $(B)$ show the results of the mapping procedure comparable to $(A)$. 

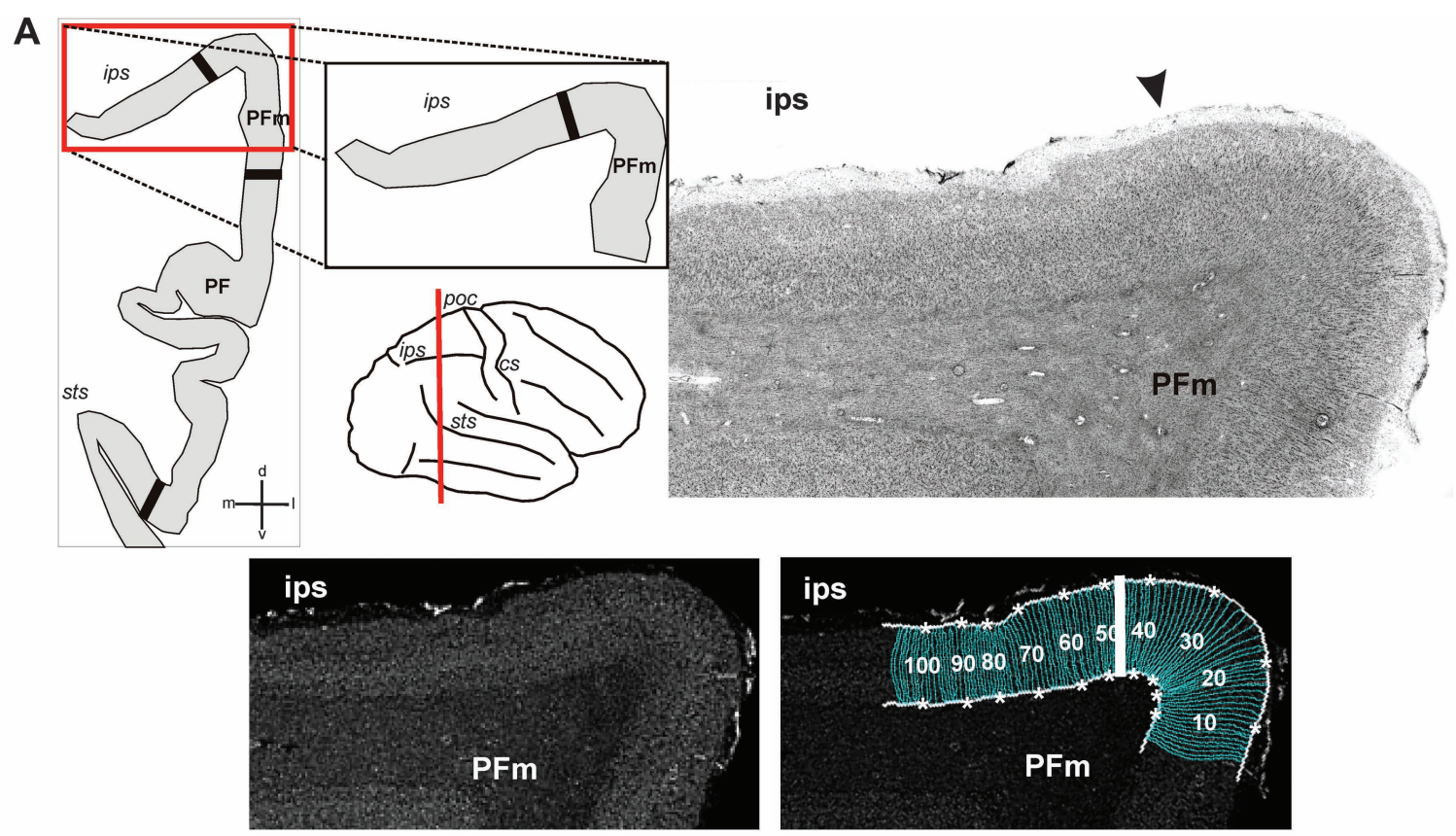

B
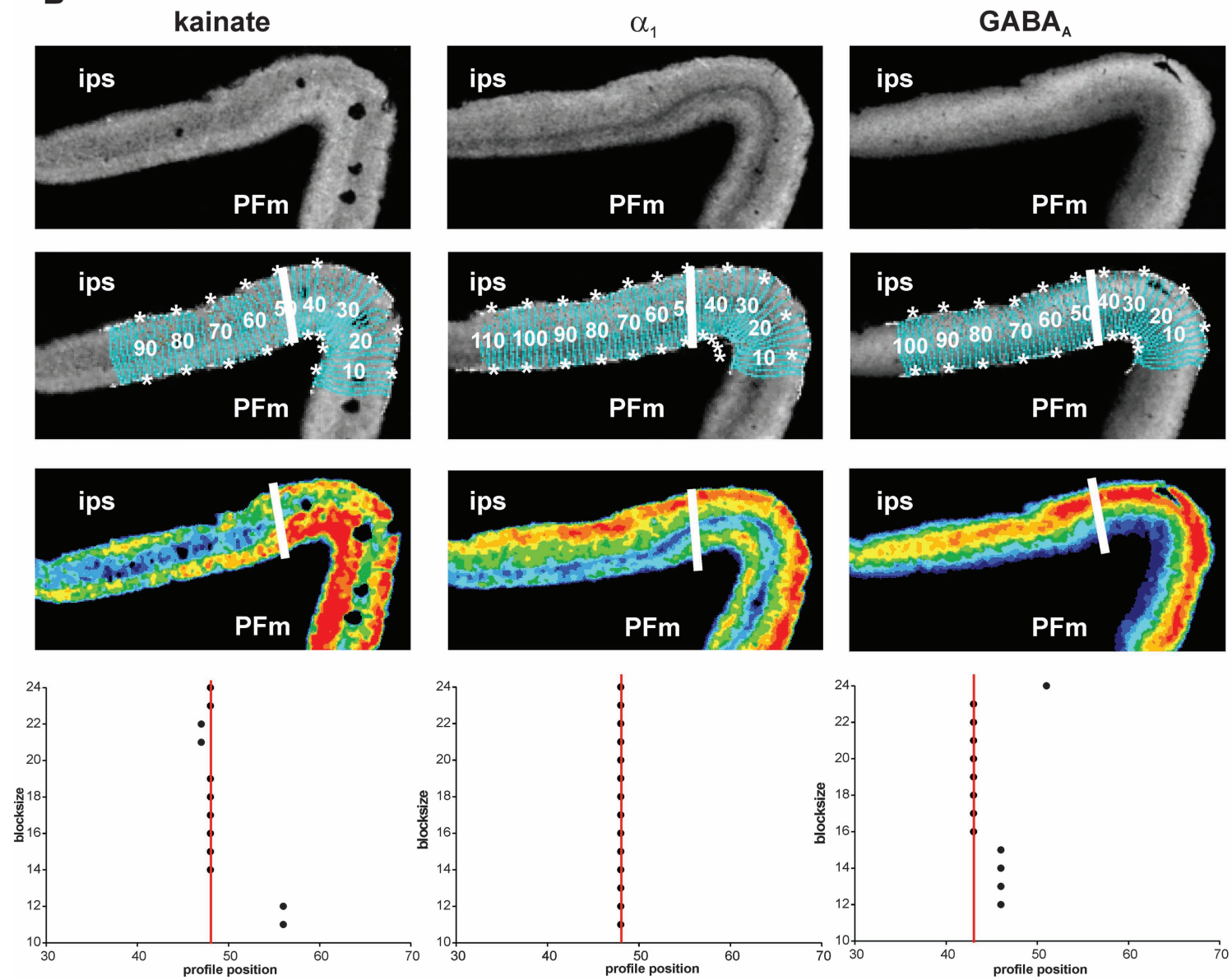

Figure 4. Algorithm-based detection of areal borders in receptor and corresponding cytoarchitectonic sections. $(A)$ Cytoarchitectonic border between area PFm and areas within the intraparietal sulcus (IPS), sectioning level (red line), and schematic drawing of the IPL within this section with all detected borders (black thick lines) depicted on the left. Corresponding gray level index image and traverses covering the cortical ribbon beneath with detected border indicated by a white bold line at profile position no. 47. (B) Same border on corresponding sections of kainate, $\mathrm{GABA}_{A}$, and $\alpha_{1}$ receptors. For each receptor, the linearized autoradiograph, superimposed with traverses covering the ROI, and pseudocolor coded for visualization purposes. Position of the border indicated by white bold lines and in the graphs at the bottom at the respective profile position (same type of graphs as in Fig. 3). Area PFm differs from intraparietal areas by means of higher concentrations of kainate in middle and lower layers, higher concentrations of $\alpha_{1}$ in infragranular layers and of $\mathrm{GABA}_{A}$ in supragranular layers. Note the close resemblance of the position of the border in cyto- and receptor sections. cs: central sulcus, ips: intraparietal sulcus, poc: postcentral sulcus, sts: superior temporal sulcus. 
The differences between the density profiles were used for the statistically testable and observer-independent definition of areal borders (for details, see Fig. 3 and Schleicher et al. 2005). As an example for the multireceptor mapping of the IPL, the receptorarchitectonically defined border for different receptor types between areas PF and PFm is shown in Figure 3.

The receptor-based parcellation approach (Zilles, PalomeroGallagher, et al. 2002; Zilles, Schleicher, et al. 2002; Morosan et al. 2005; Zilles and Amunts 2009) led to the identification of the same 7 IPL areas as previously identified by cytoarchitectonic criteria (Caspers et al. 2006, 2008): areas PFt, PFop, PF, $\mathrm{PFm}, \mathrm{PFcm}, \mathrm{PGa}$, and $\mathrm{PG}$. The precise match between receptor and cytoarchitectonic mapping can be demonstrated by comparing receptor architectonic with corresponding (neighboring) cytoarchitectonic sections of the same brain (Fig. 4).

Differences in laminar patterns largely contribute (in addition to differences in the absolute concentration within the cortex) to the regional segregation of the IPL into 7 receptor-architectonic areas. The border regions between neighboring IPL areas are shown in Figures 5 and 6.

It has already been noted that not all receptors show each border (Zilles, Palomero-Gallagher, et al. 2002; Zilles, Schleicher, et al. 2002) and that borders are not equally clear pronounced by all receptor types. However, if a border has been detected by several or all receptor types, it has the same spatial position (Figs 3-6). Differences between the rostral-most IPL areas PFop, $\mathrm{PFt}$, and PF were most prominently indicated by the kainate, NMDA, GABA $A_{\mathrm{A}}$, and $\alpha_{1}$ receptors. Here, PF showed higher concentrations for the kainate, NMDA, and $\alpha_{1}$ receptors and lower concentrations for the $\mathrm{GABA}_{\mathrm{A}}$ receptor as compared with PFt and PFop (Fig. 5).

Figure 6 displays the border regions between the more caudal IPL areas. Area PFm could be distinguished from area PF most clearly by the NMDA and $\mathrm{GABA}_{\mathrm{B}}$ receptors, whereby $\mathrm{PFm}$

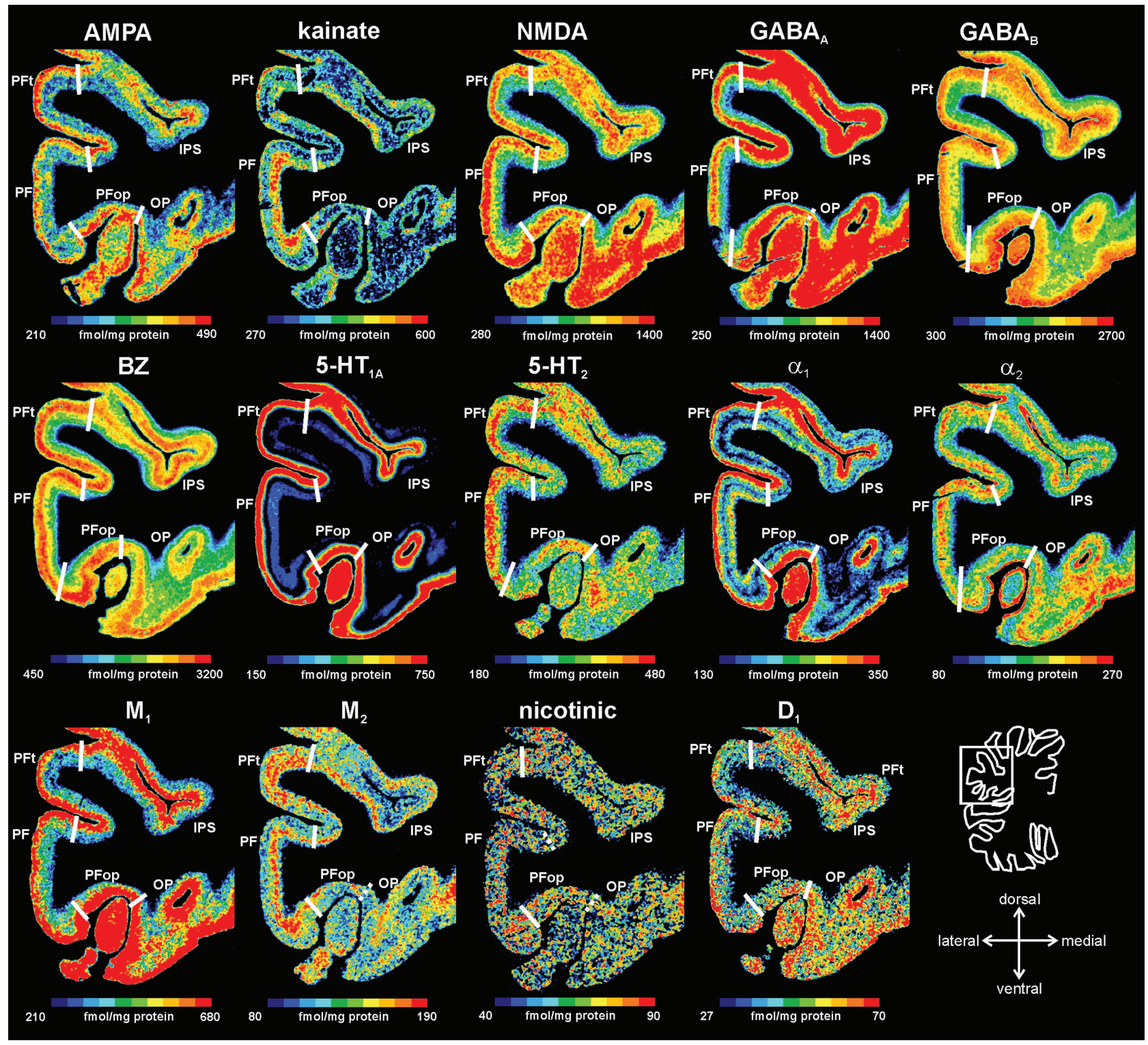

Figure 5. Receptor distribution patterns in areas PF, PFop, and PFt illustrated for 14 of the 15 receptors studied. Pseudocolor-coded autoradiographs show the borders between the IPL areas (white lines). The color bar beneath each autoradiograph indicates receptor concentrations by the different colors, from black for low to red for high concentrations (in femtomole per milligram protein). Note that the scaling is different for each receptor. 


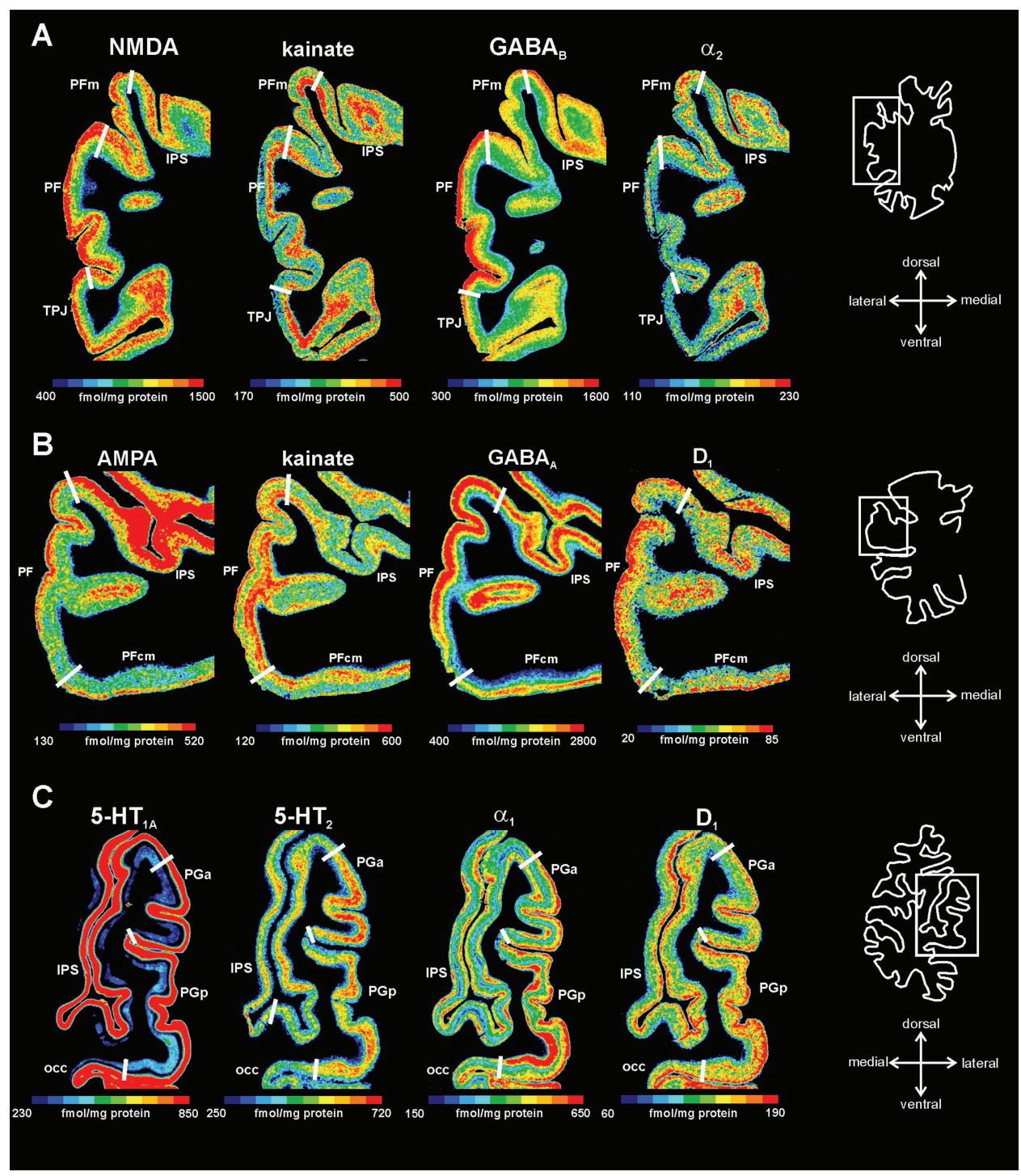

Figure 6. Receptor distribution patterns of areas PF, PFm, PFcm, PGa, and PGp for those receptors, which showed most prominent differences between the areas. (A) Delineation of areas PF and PFm (same level as in Fig. 3). (B) Delineation of areas PF and PFcm. (C) Delineation of areas PGa and PGp. For other conventions, see Figure 4.

had lower concentrations in the supragranular layers than area PF. Conversely, area PFm showed higher concentrations of kainate and $\alpha_{2}$ receptors in the supragranular layers than area PF (Fig. 6A).

Area $\mathrm{PFcm}$ most prominently differed from area $\mathrm{PF}$ with regard to the AMPA, kainate, $\mathrm{GABA}_{\mathrm{A}}$, and the $\mathrm{D}_{1}$ receptors, whereby $P F c m$ showed considerably lower concentrations than PF (Fig. 6B).

Caudal-most areas PGa and PGp were best delineated by the 5-HT $\mathrm{HA}_{1 \mathrm{~A}}, 5-\mathrm{HT}_{2}, \alpha_{1}$, and $\mathrm{D}_{1}$ receptors. PGp showed higher concentrations of $5-\mathrm{HT}_{1 \mathrm{~A}}$ and $\alpha_{1}$ receptors in the infragranular layers and of the $D_{1}$ receptor in the supragranular layers than $\mathrm{PGa}$. Concentrations of $5-\mathrm{HT}_{2}$ receptors were higher in supragranular layers of PGa as compared with PGp (Fig. 6C).

\section{Quantitative Analysis of Mean Receptor Densities}

\section{Receptor Fingerprints of the IPL Areas}

The receptor densities of each IPL area and each receptor type are displayed in Table 2. Highest mean densities (averaged over all cortical layers) are found for the NMDA, GABA,$G A B A_{B}$, and benzodiazepine-binding sites, lowest densities are reached by the $D_{1}$, nicotinic, and $M_{2}$ receptors. Maximal or minimal receptor densities of each receptor type are found in different areas of the IPL. Thus, each area has a specific balance between the different receptor types.

The area-specific balances between the 15 receptors can be visualized as "receptor fingerprints" (Fig. 7). Comparing the shapes of the fingerprints revealed a rostrocaudal gradient: The fingerprints based on absolute receptor concentrations (Fig. 7A) showed higher concentrations of the benzodiazepine binding sites in the rostral (Fig. 7, upper part) as compared with more caudal IPL areas (Fig. 7, lower part). Fingerprints based on normalized receptor concentrations (Fig. $7 B$ ) additionally showed lower AMPA, GABA,$\alpha_{2}$, and $D_{1}$ receptor concentrations and higher kainate, $5-\mathrm{HT}_{1 \mathrm{~A}}$, and $5-\mathrm{HT}_{2}$ receptor concentrations in the rostral as compared with the caudal IPL areas. Caudalmost area PGp is characterized by high concentrations of the $\mathrm{M}_{2}$ receptor, whereas area PGa shows exceptionally high concentrations of the nicotinic receptor. 


\begin{tabular}{|c|c|c|c|c|c|c|c|}
\hline \multirow[t]{2}{*}{ Receptor } & \multicolumn{7}{|c|}{ IPL areas } \\
\hline & PFop & PFt & PFcm & PF & PFm & $\mathrm{PGa}$ & PGp \\
\hline AMPA & $358.09 \pm 62.73$ & $363,65 \pm 51.11$ & $324.84 \pm 65.24$ & $424.66 \pm 65.09$ & $398.26 \pm 48,09$ & $437.99 \pm 64.62$ & $464.30 \pm 85.84$ \\
\hline Kainate & $457.37 \pm 77.49$ & $541.48 \pm 91.61$ & $521,42 \pm 131.47$ & $659.84 \pm 92.94$ & $596.46 \pm 97.96$ & $587.77 \pm 122.92$ & $487.41 \pm 104.19$ \\
\hline NMDA & $1142.85 \pm 84.49$ & $1077.73 \pm 107.60$ & $1114.54 \pm 129.36$ & $1158.25 \pm 97.03$ & $1240.96 \pm 79.20$ & $1223.37 \pm 73.86$ & $1116.08 \pm 124.75$ \\
\hline $\mathrm{GABA}_{\mathrm{A}}$ & $1588.22 \pm 131.31$ & $1675.23 \pm 126.70$ & $1460.58 \pm 164.06$ & $1508.03 \pm 124.92$ & $1539.34 \pm 102.92$ & $1939.48 \pm 157.07$ & $1832.05 \pm 244.97$ \\
\hline $\mathrm{GABA}_{\mathrm{B}}$ & $2195.31 \pm 213.24$ & $2285.14 \pm 152.90$ & $2033.04 \pm 280.58$ & $2192.64 \pm 208.12$ & $2200.67 \pm 260.67$ & $2664.09 \pm 121.44$ & $2297.44 \pm 248.66$ \\
\hline BZ & $2828.36 \pm 250.41$ & $2797.80 \pm 280.24$ & $2845.69 \pm 410.11$ & $2716.50 \pm 281.80$ & $2489.03 \pm 344.75$ & $2445.60 \pm 293.86$ & $2378.00 \pm 331.18$ \\
\hline $5-\mathrm{HT}_{1 \mathrm{~A}}$ & $439.85 \pm 73.24$ & $405.46 \pm 60.78$ & $414.99 \pm 103.43$ & $361.44 \pm 49.54$ & $335.93 \pm 47.62$ & $313.00 \pm 46.05$ & $328.45 \pm 65.46$ \\
\hline $5-\mathrm{HT}_{2}$ & $433.94 \pm 41.13$ & $434.17 \pm 48.31$ & $434.28 \pm 53.21$ & $441.92 \pm 49.34$ & $424.76 \pm 59.87$ & $412.24 \pm 70.71$ & $382.79 \pm 59.43$ \\
\hline$M_{1}$ & $535.57 \pm 73.88$ & $470.10 \pm 61.39$ & $497.67 \pm 92.89$ & $459.90 \pm 69.93$ & $489.62 \pm 80.42$ & $456.47 \pm 67.90$ & $452.02 \pm 61.00$ \\
\hline $\mathrm{M}_{2}$ & $159.29 \pm 14.33$ & $161.32 \pm 12.12$ & $138.68 \pm 19.31$ & $170.53 \pm 25.40$ & $173.50 \pm 26.22$ & $158.87 \pm 29.98$ & $201.75 \pm 48.54$ \\
\hline$M_{3}$ & $902.58 \pm 220.18$ & $850.92 \pm 178.10$ & $660.20 \pm 143.45$ & $821.06 \pm 105.22$ & $775.76 \pm 107.59$ & $736.76 \pm 96.82$ & $741.39 \pm 131.85$ \\
\hline nic & $44.93 \pm 10.09$ & $48.10 \pm 8.83$ & $38.29 \pm 8.85$ & $58.91 \pm 13.44$ & $60.39 \pm 11.46$ & $68.50 \pm 11.51$ & $46.07 \pm 11.59$ \\
\hline$\alpha_{1}$ & $362.04 \pm 36.82$ & $365.44 \pm 34.94$ & $335.63 \pm 36.52$ & $372.21 \pm 57.03$ & $393.38 \pm 49.38$ & $343.41 \pm 50.17$ & $356.91 \pm 40.37$ \\
\hline$\alpha_{2}$ & $304.26 \pm 80.51$ & $335.93 \pm 88.95$ & $297.52 \pm 95.33$ & $370.43 \pm 118.34$ & $303.08 \pm 84.95$ & $343.25 \pm 86.58$ & $327.10 \pm 64.74$ \\
\hline$D_{1}$ & $89.11 \pm 10.07$ & $81.54 \pm 11.86$ & $86.46 \pm 12.34$ & $100.46 \pm 15.63$ & $105.61 \pm 16.27$ & $132.44 \pm 18.00$ & $105.14 \pm 16.55$ \\
\hline
\end{tabular}

Note: Fifteen different receptors were measured in 9 hemispheres. SD: standard deviation.

\section{Molecular Organization of the IPL}

For a comprehensive analysis of similarities between the receptor fingerprints of the different IPL areas, we performed a hierarchical cluster analysis (Fig. 8A). Three groups with similar receptor distributions within each group were identified: a rostroventral group with areas PFt, PFop, and PFcm; a middle group of areas $\mathrm{PF}$ and $\mathrm{PFm}$; and a caudal group consisting of areas PGa and PGp. Furthermore, it became apparent that the PG areas were more similar to each other as compared with the rest of the IPL. This result reflects a clear architectural distinction between rostral and caudal IPL.

A consecutive canonical discriminant analysis with 2 discriminating dimensions ( $x$ - and $y$-axes in Fig. $8 B$ ) revealed a most pronounced distinction between the clusters within the first dimension ( $x$-axis; $72 \%$ explained variance), complemented by the distinction within the second dimension ( $y$-axis; 28\% explained variance). Ranking the coefficients of the canonical discriminant analysis revealed those receptors, which contributed most to the distinction between the clusters in both dimensions. The kainate and $5-\mathrm{HT}_{2}$ receptors contributed most to the distinction in both dimensions (absolute values of the coefficients: kainate: 2.22 [score 1] and 1.91 [score 2]; $5-\mathrm{HT}_{2}$ : 2.84 [score 1] and 1.58 [score 2]). The $\mathrm{M}_{1}$ and $\alpha_{2}$ receptors provided additional criteria for this segregation within the first dimension (absolute values of coefficients: $M_{1}: 1.10 ; \alpha_{2}: 1.05$ ), whereas the $G_{A B A}$, nicotinic, and $D_{1}$ receptors were responsible for distinction between the clusters within the second dimension (absolute values of coefficients: $\mathrm{GABA}_{\mathrm{A}}$ : 1.02 ; nicotinic: $1.10 ; \mathrm{D}_{1}$ : 1.18).

MDS analysis (Fig. 8C) of the receptor densities highlights an inhomogeneity within the caudal cluster of areas PGa and PGp: the receptor organization of $\mathrm{PGp}$ seems to be more different (higher distance) from all the other IPL areas. This dissimilarity was not revealed by our previous cytoarchitectonic analysis of the IPL (Caspers et al. 2006, 2008). Based on the present results, area PGp might be reclassified as not being a typical parietal cortex. It might provide a transition to adjoining visual cortex, which can be underpinned by comparison with the receptor architecture of other cortical areas (see next paragraph).

\section{Comparison with Other Cortical Areas}

We compared the IPL fingerprints with those of primary, secondary, and higher order sensory areas and the motor cortex to study the functional aspect of the receptor-based IPL segregation (Fig. 9).

Using a hierarchical cluster analysis, the fingerprints of the primary and secondary auditory and visual as well as primary somatosensory and motor cortices differed considerably from the cluster containing the IPL areas. The 7 IPL areas formed the same rostral, middle, and caudal subclusters as already found in the first cluster analysis of the IPL areas alone (Fig. 8A-C). The middle and caudal clusters of IPL areas are more similar to area 44 of Broca's area than the rostral cluster comprising PFcm, PFop, and PFt. The fingerprints of the centrally positioned areas $\mathrm{PF}$ and PFm are similar to the fingerprint of the superior parietal lobule. The caudally positioned areas PGa and PGp are similar to the higher ventral extrastriate area hOC3v (V3v), particularly for area PGp.

This result suggests again a potential role of area PGp as a higher visual area, linking occipital and parietal cortex.

\section{Discussion}

Functional performance of a cortical area depends on a welltuned and area-specific balance between numerous receptor types (Barnes and Sharp 1999; Goldman-Rakic et al. 2000; Gibbs and Summers 2002; Bergson et al. 2003; Bredt and Nicoll 2003; Friedman et al. 2004). Based on the similarities in receptor fingerprints of the 7 IPL areas, we propose a new organizational model of the IPL (Fig. 8D), comprising a rostroventral (areas PFt, PFop, and PFcm), an intermediate (areas PF and PFm), and a caudal group (areas PGa and PGp). The molecular structure of caudal-most IPL area PGp argues for a reclassification of this area as transition area between parietal and visual areas.

\section{The 3-Region Model of Human IPL}

It has been shown repeatedly that receptor distributions are not only related to functional network properties of cortical areas (Barnes and Sharp 1999; Goldman-Rakic et al. 2000; Gibbs and Summers 2002; Bergson et al. 2003; Bredt and Nicoll 2003; Friedman et al. 2004) but also to their connectivity pattern (Rakic et al. 1988). The 3-region model of human IPL as revealed by multireceptor distribution could thus provide the molecular basis for the structural, functional, and connectivity components within a common organizational framework. 


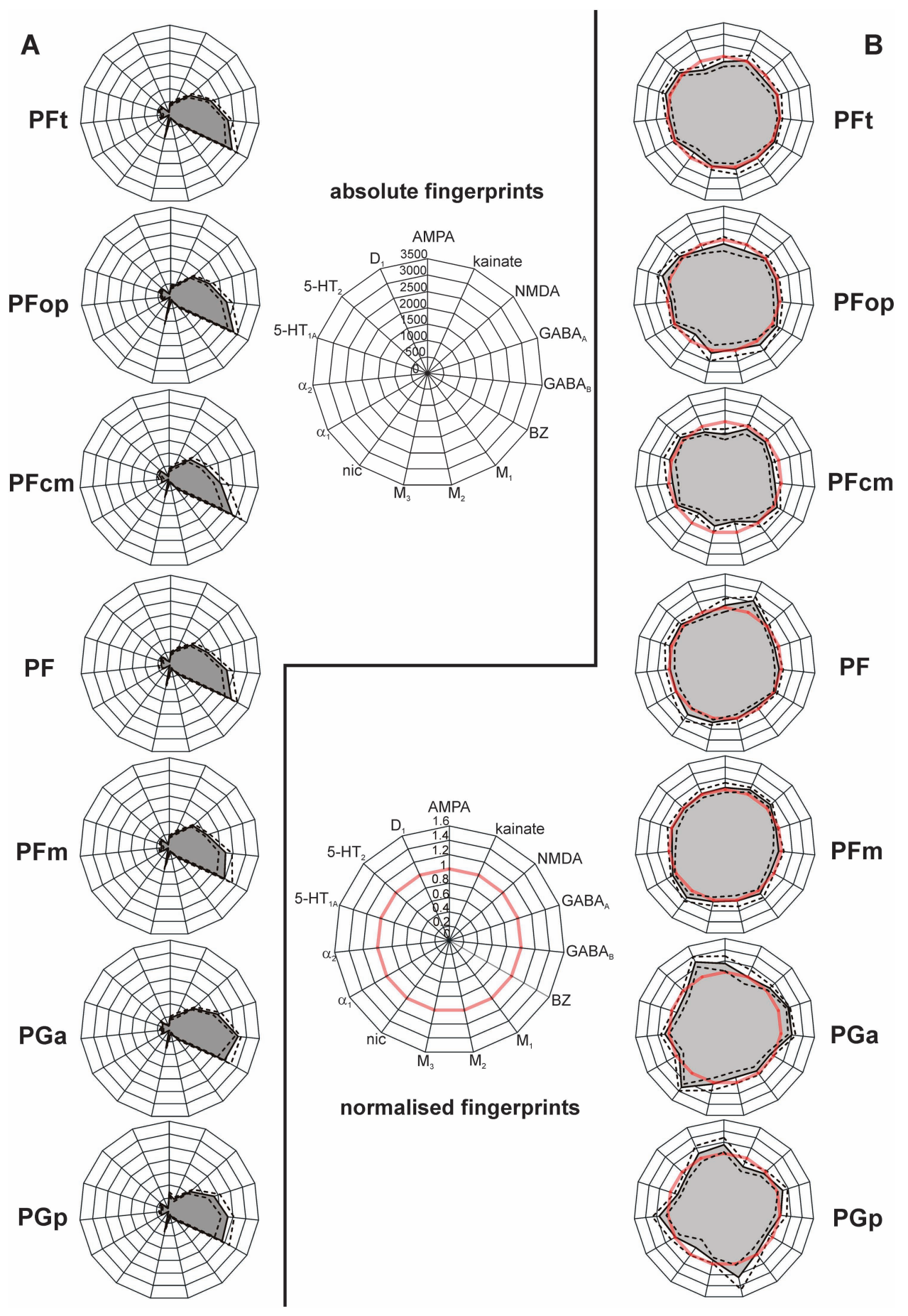

Figure 7. Receptor fingerprints of the $7 \mathrm{IPL}$ areas PFt, PFop, PF, PFm, PFcm, PGa, and PGp. (A) Polar plots (scaling 0-3500 femtomole per milligram protein) showing the mean (averaged over all cortical layers) absolute receptor concentrations of all 15 receptors (with standard error of the mean as dotted lines) of each area. (B) Polar plots (scaling 0 1.6) showing the normalized receptor concentration of all 15 receptors (with standard error of the mean as dotted lines). Normalization of the receptor concentrations was calculated based on each receptor's mean over the whole IPL. Red thick line indicates the 100\% line (labeled 1) where the receptor concentration of an area was equal to the mean receptor concentration averaged over the whole IPL. Note the difference in size and shape between the fingerprints of the different areas.

\section{Fiber Tracts}

The fiber tracts of the IPL show pronounced differences between rostral, middle, and caudal IPL. The rostral IPL is most likely connected with ventral premotor cortex and the caudal IPL with temporal areas (Croxson et al. 2005; Rushworth et al. 2006; Tomassini et al. 2007). This finding was supported by resting-state functional connectivity and structural connectivity- based parcellation analyses (Mars et al. 2011), which demonstrated a subdivision of right human IPL into 5 clusters, largely matching the cytoarchitectonic subdivision (Caspers et al. 2006, 2008). Caspers, Eickhoff, et al. (2011) demonstrated in a probabilistic fiber tracking study that rostral IPL areas had connections with inferior and middle frontal, premotor, primary motor, and somatosensory areas, whereas the connections of the more 
A

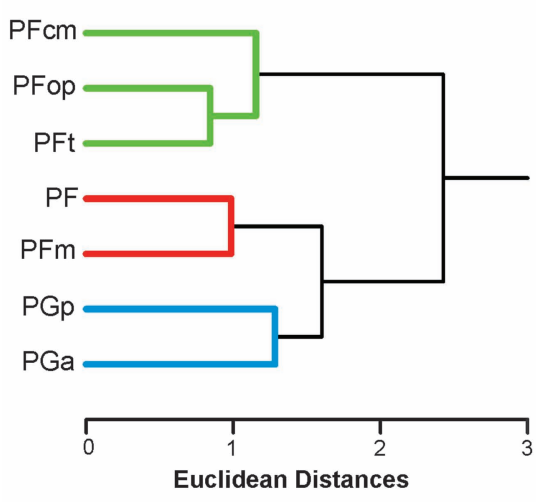

B Discriminant analysis

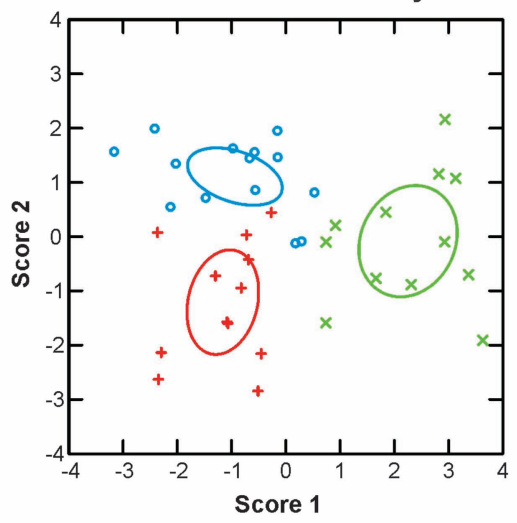

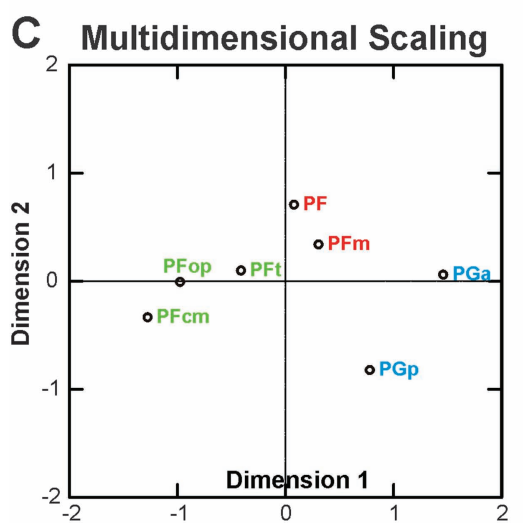

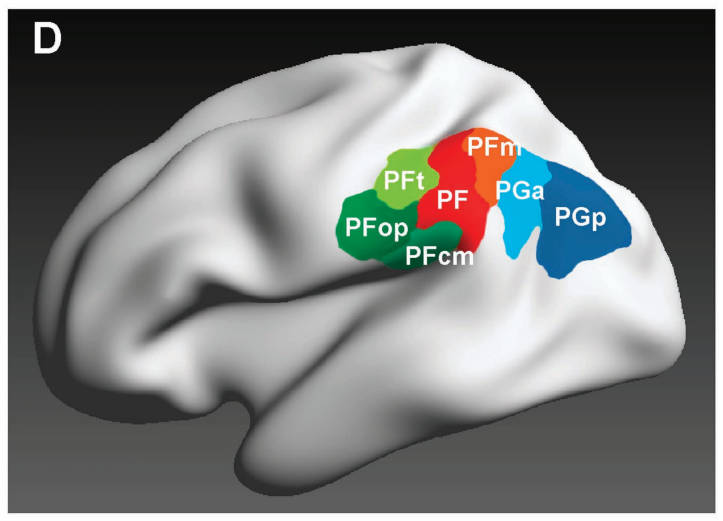

Figure 8. Segregation of IPL areas based on multiple receptor densities averaged over all cortical layers. (A) Hierarchical cluster analysis reveals 3 receptor-architectonically distinct clusters: a rostral cluster with areas PFop, PFt, PFcm (green), an intermediate cluster with areas PF and PFm (red), and a caudal cluster with areas PGa and PGp (blue). (B) Canonical discriminant analysis of all available receptor data in IPL. For each of the 3 clusters, the $n$ data points ( $n=$ number of areas in that cluster $\times$ number of hemispheres, some points are missing due to missing values for some receptor types) are indicated by different symbols. Ellipses provide the $90 \%$ confidence interval of the centroids. Same color coding as in $(A)$. $(C)$ MDS analysis visualizes the differences between the 3 clusters. Same color coding as in $(A)$. (D) Visualization of the resulting 3 clusters within the IPL, using the same depiction of the cytoarchitectonically defined IPL areas (Caspers et al. 2006, 2008) as in Figure 1. Color coding of the areas corresponding to the receptor-based cluster segregation: rostral cluster (areas PFt, PFop, and PFcm): shades of green; middle cluster (areas PF and PFm): shades of red; caudal cluster (areas PGa and PGp): shades of blue.

central and caudal IPL areas shifted to target regions in superior parietal, extrastriate visual, and temporal cortices. The middle areas shared connection patterns of both rostral and caudal areas with prominent connections to frontal, superior parietal, and intraparietal areas. The differences in connection patterns between rostral, middle (sharing connection patterns of rostral and caudal IPL areas), and caudal IPL areas thus favored the view of a tripartition of the cortex in the IPL.

\section{Functions}

Shalom and Poeppel (2008) proposed such a tripartition for the involvement of the IPL in language tasks. The IPL was assumed to provide the analysis part within the larger language framework comprising frontal, parietal, and temporal cortices. Different functional aspects of language are processed in each of the 3 partitions of these regions. In the IPL, the rostral partition processes sounds and single phonemes, that is, the basic components of language; middle IPL areas provide the syntax, that is, the rule which needs to be applied to assemble the basic components; caudal IPL areas finally determine the semantic content of words or sentences and thus refer to the meaning of information.

The receptor balance of the middle and caudal IPL areas strongly resembled that of Broca's region, whereas the finger- prints of the rostral cluster were less similar. A relationship of middle and caudal IPL areas with the core of Broca's region was also found in a recent study on fiber tracts of the IPL areas (Caspers, Eickhoff, et al. 2011) where middle and caudal IPL areas were most likely connected with Broca's region. This connectivity study and our receptor patterns fit well the model proposed by Shalom and Poeppel (2008) suggesting different and comparable hierarchical levels of language processing within the frontal and inferior parietal lobe: rostral IPL was supposed to share functional properties with ventral premotor cortex, caudally adjacent to the Broca region (area 44), whereas middle and caudal IPL areas strongly interact with Broca's region to fulfill the "rule application function" and "analysis of meaning function." Receptor-architectonic, functional, and connectivity data thus provide evidence for a 3-region model within the language domains of the frontal and parietal lobes. Thus, the 3-region model of the IPL suggests a new organizational principle in this brain region.

Functional neuroimaging studies of the IPL using other than language-related tasks further support the proposal of a tripartite organization. Rostral human IPL areas were found to be involved in reaching and grasping tasks (Peeters et al. 2009). The very rostral part was found to be activated during observation of tool use and interpreted as being uniquely human. 


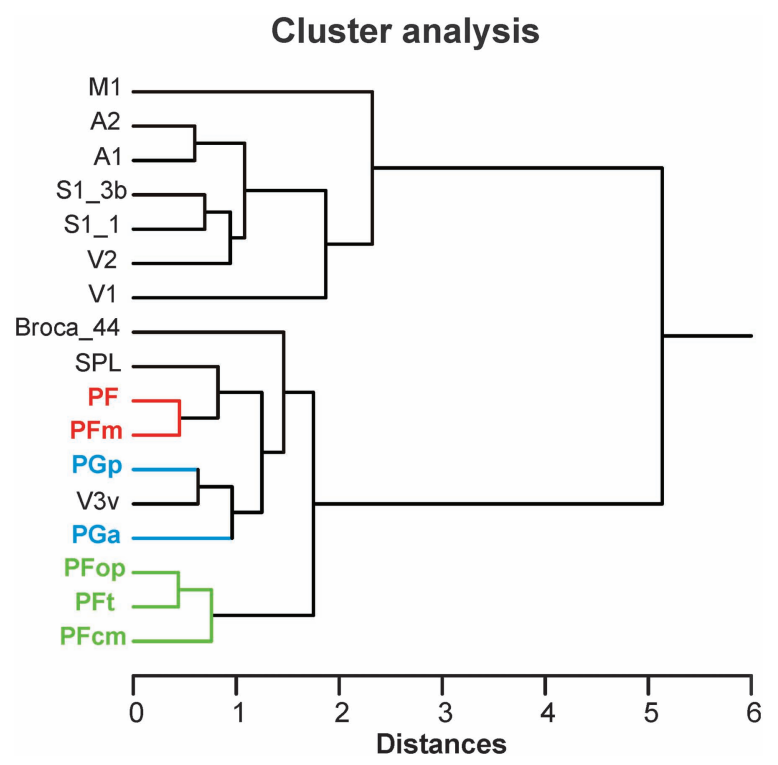

Figure 9. Receptor distributions of IPL areas compared with those of other cortical areas. The hierarchical cluster analysis that shows the same tripartition of the IPL areas as shown in Figure 8 but additionally reveals similarities of the intermediate cluster (areas PF and PFm, red) with superior parietal areas (SPLs) and of the caudal cluster (areas PGa and PGp, blue) with extrastriate visual areas. The IPL areas are most similar to each other and similar to higher order areas (Broca 44, SPL, and V3v) but are most dissimilar to primary and secondary areas (A1/A2, M1, S1, and V1/N2). Note the close resemblance of area PGp with extrastriate visual area V3v. A1/A2: primary/secondary auditory cortex, Broca_44: area 44 of Broca's region, M1: primary motor cortex, S1_3b: area 3b of primary somatosensory cortex, S1_1: area 1 of primary somatosensory cortex, SPL: superior parietal lobule, V1 $\bar{N} 2$ : primary/ secondary visual cortex, V3v: ventral extrastriate visual cortex.

Recent meta-analyses demonstrated that rostral IPL area PFt participates in the action observation and imitation network (Molenberghs et al. 2009; Van Overwalle and Baetens 2009; Caspers et al. 2010). Data from studies in macaques also point to the relevance of rostral-most IPL together with ventral premotor area 6 for the mirror neuron system (Rizzolatti 2005; Petrides and Pandya 2009). This fits the interaction within the language network (Shalom and Poeppel 2008) as described above. The middle IPL areas PF and PFm are activated by nonspatial attention tasks, especially when reevaluating conflicting choice options (Vossel et al. 2006; Boorman et al. 2009; Mevorach et al. 2009; Caspers, Heim, et al. 2011) as well as spatial attention and reorienting tasks (Rushworth et al. 2001; Corbetta et al. 2008). Together with intraparietal areas, middle IPL contributes to rule change during visually guided attention (Corbetta and Shulman 2002). Caudal areas PGa and PGp were most prominently implicated in language-related processing with special focus on semantic and phonological processing, partially found in both hemispheres (Price 2000; Hickok and Poeppel 2004; Marangolo et al. 2006; Vigneau et al. 2006). These areas have also consistently been found during moral decision making, being particularly concerned with egocentric and allocentric perspective taking (for review: Raine and Yang 2006).

The involvement of the IPL within different functional domains could thus be summarized as follows: Rostral IPL deals with tool, action, or sound. Middle IPL areas provide rules for word differentiation as well as visually guided attention and nonspatial attention processes. Caudal IPL is involved in decoding the meaning of words, scenes, or personal morally relevant interactions. Thus, the same IPL areas are involved in different tasks, which should have a functional commonality representing the role of the IPL areas on a more abstract level. It already seems plausible to assume that the hierarchical 3-region model of language functions in the IPL is a starting point for searching analogous commonalities in other functional domains.

The present study provides evidence for a general 3-region model of the IPL on a molecular basis regarding the receptor balance of different neurotransmitter systems. The relevance of the receptor balance of an area for its involvement in different functional networks has been repeatedly stressed (Barnes and Sharp 1999; Goldman-Rakic et al. 2000; Gibbs and Summers 2002; Bergson et al. 2003; Bredt and Nicoll 2003; Friedman et al. 2004). It can thus be assumed that not the distribution pattern of a single receptor, but the interplay between different receptors of different neurotransmitter systems as displayed in the receptor fingerprints of each IPL area (Fig. 7) might set the molecular basis for the role, which is played by 3 different parts of the IPL across various functional domains.

\section{The Role of Area PGp}

The present findings additionally provide new insights into the potential role of area PGp. Its receptor distribution was different from the other IPL areas and showed most pronounced similarities with higher extrastriate visual areas, particularly $\mathrm{V} 3 \mathrm{v}$. This is further promoted by connectivity analyses, which showed consistent connections between PGp and extrastriate visual areas (Caspers, Eickhoff, et al. 2011). It might thus be hypothesized that area PGp might serve as linking hub between occipital and parietal cortex for transformation of visual input to visual associations.

The cytoarchitectonic analysis of the IPL areas (Caspers et al. 2006) did not show a comparable difference. The cytoarchitecture of area PGp resembled that of the other IPL areas. It could be clearly demarcated from areas of the occipital cortex where the layers are dominated by large pyramidal cells as described by von Economo and Koskinas (1925). Area PGp is, therefore, clearly different from this "occipital type" of cortical architecture at the cytoarchitectonical level.

The probabilistic fiber tracking with area PGp as seed region shows connections to extrastriate visual areas (Caspers, Eickhoff, et al. 2011). The same situation was demonstrated in macaques for area Opt (Cavada and Goldman-Rakic 1989a, 1989b; Andersen et al. 1990; Rozzi et al. 2006), which favored the view that visual input to the IPL arrives via this caudal-most area.

The visual system has classically been subdivided into a ventral and dorsal visual stream, processing either "what" or "where" information, respectively (Ungerleider and Mishkin 1982; Ungerleider and Haxby 1994). The role of the dorsal visual stream within this framework was nevertheless not fully elucidated, fostering the notion of not only processing "where" but "how" information (Goodale and Milner 1992; Milner and Goodale 1995; Kravitz et al. 2011). It was furthermore suggested that the 2 systems are not fully separated from each other but rather interact to fulfill the task of providing the information on how an action should be executed (Pisella et al. 2006; Kravitz et al. 2011). The interaction is supposed to involve a ventrodorsal pathway, which involves caudal IPL. This region interacts with medially located areas of the superior parietal lobule, the posterior cingulate and retrosplenial cortex, and the parahippocampal gyrus. It might provide information about peripersonal 
space with regard to egocentric or allocentric perspectives (Pisella et al. 2006; Rushworth et al. 2006; Kravitz et al. 2011). This corresponds to the findings of activation within caudal IPL during moral decision making where additional activation clusters were found in posterior cingulate cortex in addition to ventral and medial prefrontal cortex (Raine and Yang 2006). Here, the simultaneous activation of caudal IPL with posterior cingulate cortex was especially found during personal versus impersonal and utilitarian versus nonutilitarian moral judgments (Greene et al. 2004). Both these decisions involve allocentric versus egocentric perspectives to come to the respective moral judgment.

The results of the present study support the idea of area PGp serving as higher visual processing hub within the IPL. The similarity between the receptor balances of area PGp with that of ventral extrastriate visual area hOC3v (V3v) supports the idea that area PGp is key region in the ventrodorsal visual stream (Pisella et al. 2006), since it receives input from an area of that visual stream.

\section{Conclusions and Outlook}

Based on the regionally specific multireceptor balances (receptor fingerprints), a 3-region model of human IPL is proposed (Fig. 8D). A hierarchical cluster analysis of the receptor fingerprints between the IPL areas and visual-, motor-, auditory-, and language-related cortical areas shows the highest similarity of all IPL areas with area 44 of Broca's region, of the areas in the middle of IPL with the superior parietal cortex, and for the most caudal areas with the extrastriate visual cortex. Notably, PGp has a receptor fingerprint very similar to that of ventral extrastriate area hOC3v (V3v). Since receptor fingerprints covary with the cytoarchitecture, function, and connectivity of each IPL area, the present study provides a molecular perspective of the organizational principles behind the regional and functional segregation of the IPL.

As a link to the function of an area, the receptor-based delineation of cortical areas poses an additional question: Do the receptor density patterns always follow the cytoarchitectonic boundaries? In our study, we independently mapped receptor profiles of each receptor and defined receptor-based borders within the respective sections. Additionally, we measured the cytoarchitectonic profiles of the same brain within alternate cell body-stained sections. The borders of both approaches did precisely coincide. It has to be noted that not all receptors showed every border of the IPL or other cortical areas (Zilles, Palomero-Gallagher, et al. 2002; Amunts et al. 2010). Vice versa, some receptors might show additional borders, which would hint at further subdivisions of cortical areas on a molecular level. Taking an independent mapping approach for all receptors will allow providing complete brain maps for each receptor in future studies, each revealing an individual view on the molecular architecture of the cortex.

\section{Supplementary Material}

Supplementary material can be found at: http://www.cercor. oxfordjournals.org/

\section{Funding}

This project was supported by grants of the Initiative and Networking Fund of the Helmholtz Association within the
Helmholtz Alliance on Systems Biology (Human Brain Model to K.Z.); the Helmholtz Alliance for Mental Health in an Aging Society (HelMA to K.A. and K.Z.); and the German Ministry for Education and Research (01GW0771 and 01GW0623 to K.A.). The funders had no role in study design, data collection and analysis, decision to publish, or preparation of the manuscript.

\section{Notes}

Conflict of Interest: None declared.

\section{References}

Amunts K, Lenzen M, Friederici AD, Schleicher A, Morosan P, PalomeroGallagher N, Zilles K. 2010. Broca's region: novel organizational principles and multiple receptor mapping. PLoS Biol. 8(9):e1000489.

Amunts K, Malikovic A, Mohlberg H, Schormann T, Zilles K. 2000. Brodmann's areas 17 and 18 brought into stereotaxic space-where and how variable? Neuroimage. 11:66-84.

Amunts K, Schleicher A, Bürgel U, Mohlberg H, Uylings HBM, Zilles K. 1999. Broca's region revisited: cytoarchitecture and intersubject variability. J Comp Neurol. 412:319-341.

Andersen RA, Asanuma C, Essick G, Siegel RM. 1990. Corticocortical connections of anatomically and physiologically defined subdivisions within the inferior parietal lobule. J Comp Neurol. 296:65-113.

Barnes NM, Sharp T. 1999. A review of central 5-HT receptors and their function. Neuropharmacology. 38(8):1083-1152.

Bergson C, Levenson R, Goldman-Rakic PS, Lidow MS. 2003. Dopamine receptor-interacting proteins: the $\mathrm{Ca}(2+)$ connection in dopamine signalling. Trends Pharmacol Sci. 24:486-492.

Boorman ED, Behrens TE, Woolrich MW, Rushworth MF. 2009. How green is the grass on the other side? Frontopolar cortex and the evidence in favor of alternative courses of action. Neuron. 62(5):733-743.

Bredt DS, Nicoll RA. 2003. AMPA receptor trafficking at excitatory synapses. Neuron. 40:361-379.

Brodmann K. 1909. Vergleichende Lokalisationslehre der Großhirnrinde. Leipzig (Germany): Barth.

Caspers S, Eickhoff SB, Geyer S, Scheperjans F, Mohlberg H, Zilles K, Amunts K. 2008. The human inferior parietal lobule in stereotaxic space. Brain Struct Funct. 212:481-495.

Caspers S, Eickhoff SB, Rick T, von Kapri A, Kuhlen T, Huang R, Shah NJ, Zilles K. 2011. Probabilistic fibre tract analysis of cytoarchitectonically defined human inferior parietal lobule areas reveals similarities to macaques. Neuroimage. 58(2):362-380.

Caspers S, Geyer S, Schleicher A, Mohlberg H, Amunts K, Zilles K. 2006. The human inferior parietal cortex: cytoarchitectonic parcellation and interindividual variability. Neuroimage. 33(2):430-448.

Caspers S, Heim S, Lucas MG, Stephan E, Fischer L, Amunts K, Zilles K. 2011. Moral concepts set decision strategies to abstract values. PLoS One. 6(4):e18451.

Caspers S, Zilles K, Laird AR, Eickhoff SB. 2010. ALE meta-analysis of action observation and imitation in the human brain. Neuroimage. 50:1148-1167.

Cavada C, Goldman-Rakic PS. 1989a. Posterior parietal cortex in rhesus monkey: I. Parcellation of areas based on distinctive limbic and sensory corticocortical connections. J Comp Neurol. 287:393-421.

Cavada C, Goldman-Rakic PS. 1989b. Posterior parietal cortex in rhesus monkey: II. Evidence of segregated corticocortical networks linking sensory and limbic areas with the frontal lobe. J Comp Neurol. 287:422-445.

Corbetta M, Patel G, Shulman GL. 2008. The reorienting system of the human brain: from environment to theory of mind. Neuron. 58:306-324.

Corbetta M, Shulman GL. 2002. Control of goal-directed and stimulusdriven attention in the brain. Nat Rev Neurosci. 3(3):201-215.

Croxson PL, Johansen-Berg H, Behrens TE, Robson MD, Pinsk MA, Gross CG, Richter W, Richter MC, Kastner S, Rushworth. 2005. Quantitative investigation of connections of the prefrontal cortex in the human and macaque using probabilistic diffusion tractography. J Neurosci. 25:8854-8866. 
Eickhoff SB, Rottschy C, Kujovic M, Palomero-Gallagher N, Zilles K. 2008. Organizational principles of human visual cortex revealed by receptor mapping. Cereb Cortex. 18(11):2637-2645.

Eickhoff SB, Rottschy C, Zilles K. 2007. Laminar distribution and codistribution of neurotransmitter receptors in early human visual cortex. Brain Struct Funct. 212:255-267.

Fink GR, Marshall JC, Weiss PH, Zilles K. 2001. The neural basis of vertical and horizontal line bisection judgements: an fMRI study of normal volunteers. Neuroimage. 14:59-67.

Fogassi L, Ferrari PF, Gesierich B, Rozzi S, Chersi F, Rizzolatti G. 2005. Parietal lobe: from action organization to intention understanding. Science. 308:662-667.

Friedman JI, Stewart DG, Gorman JM. 2004. Potential noradrenergic targets for cognitive enhancement in schizophrenia. CNS Spectr. 9:350-355.

Geyer S, Ledberg A, Schleicher A, Kinomura S, Schormann T, Bürgel U, Klingberg T, Larsson J, Zilles K, Roland PE. 1996. Two different areas within the primary motor cortex of man. Nature. 382:805-807.

Geyer S, Matelli M, Luppino G, Schleicher A, Jansen Y, PalomeroGallagher N, Zilles K. 1998. Receptor autoradiographic mapping of the mesial motor and premotor cortex of the macaque monkey. J Comp Neurol. 397:231-250.

Geyer S, Schleicher A, Zilles K. 1997. The somatosensory cortex of human: cytoarchitecture and regional distributions of receptorbinding sites. Neuroimage. 6:27-45.

Geyer S, Schleicher A, Zilles K. 1999. Areas 3a, 3b, and 1 of human primary somatosensory cortex: 1 . Microstructural organization and interindividual variability. Neuroimage. 10(1):63-83.

Geyer S, Schormann T, Mohlberg H, Zilles K. 2000. Areas 3a, 3b, and 1 of human primary somatosensory cortex. Part 2: spatial normalization to standard anatomical space. Neuroimage. 11:684-696.

Gibbs ME, Summers RJ. 2002. Role of adrenoceptor subtypes in memory consolidation. Prog Neurobiol. 67(5):345-391.

Goldman-Rakic PS, Muly EC III, Williams GV. 2000. D(1) receptors in prefrontal cells and circuits. Brain Res Brain Res Rev. 31:295-301.

Goodale MA, Milner AD. 1992. Separate visual pathways for perception and action. Trends Neurosci. 15:20-25.

Greene JD, Nystrom LE, Engell AD, Darley JM, Cohen JD. 2004. The neural bases of cognitive conflict and control in moral judgment. Neuron. 44:389-400.

Gregoriou GG, Borra E, Matelli M, Luppino G. 2006. Architectonic organization of the inferior parietal convexity of the macaque monkey. J Comp Neurol. 496:422-451.

Hickok G, Poeppel D. 2004. Dorsal and ventral streams: a framework for understanding aspects of the functional anatomy of language. Cognition. 92:67-99.

Hyvärinen J. 1982. Posterior parietal lobe of the primate brain. Physiol Rev. 62:1060-1129.

Iacoboni M. 2005. Neural mechanisms of imitation. Curr Opin Neurobiol. 15:632-637.

Keysers C, Gazzola V. 2009. Expanding the mirror: vicarious activity for actions, emotions, and sensations. Curr Opin Neurobiol. 19:666-671.

Kravitz DJ, Kadharbatcha SS, Baker CI, Mishkin M. 2011. A new neural framework for visuospatial processing. Nat Rev Neurosci. 12:217-230.

Marangolo P, Piras F, Galati G, Burani C. 2006. Functional anatomy of derivational morphology. Cortex. 42:1093-1106.

Mars RB, Jbabdi S, Sallet J, O'Reilly JX, Croxson PL, Olivier E, Noonan MP, Bergmann C, Mitchell AS, Baxter MG, et al. 2011. Diffusion-weighted imaging tractography-based parcellation of the human parietal cortex and comparison with human and macaque resting-state functional connectivity. J Neurosci. 31(11):4087-4100.

Merker B. 1983. Silver staining of cell bodies by means of physical development. J Neurosci Methods. 9:235-241.

Mevorach C, Humphreys GW, Shalev L. 2009. Reflexive and preparatory selection and suppression of salient information in the right and left posterior parietal cortex. J Cogn Neurosci. 21:1204-1214.

Milner AD, Goodale MA. 1995. The visual brain in action. Oxford: Oxford University Press.
Molenberghs P, Cunnington R, Mattingley JB. 2009. Is the mirror neuron system involved in imitation? A short review and metaanalysis. Neurosci Biobehav Rev. 33(7):975-980.

Morosan P, Rademacher J, Schleicher A, Amunts K, Schormann T, Zilles K. 2001. Human primary auditory cortex: cytoarchitectonic subdivisions and mapping into a spatial reference system. Neuroimage. 13:684-701.

Morosan P, Schleicher A, Amunts K, Zilles K. 2005. Multimodal architectonic mapping of human superior temporal gyrus. Anat Embryol (Berl). 210(5-6):401-406.

Mountcastle VB, Lynch JC, Georgopoulos A, Sakata H, Acuna C. 1975. Posterior parietal association cortex of the monkey: command functions for operations within extrapersonal space. J Neurophysiol. 38:871-908.

Palomero-Gallagher N, Vogt BA, Schleicher A, Mayberg HS, Zilles K. 2009. Receptor architecture of human cingulate cortex: evaluation of the four-region neurobiological model. Hum Brain Mapp. 30(8):2336-2355.

Pandya DN, Seltzer B. 1982. Intrinsic connections and architectonics of posterior parietal cortex in the rhesus monkey. J Comp Neurol. 204:196-210.

Peeters R, Simone L, Nelissen K, Fabbri-Destro M, Vanduffel W, Rizzolatti G, Orban GA. 2009. The representation of tool use in humans and monkeys: common and uniquely human features. J Neurosci. 29(37):11523-11539.

Petrides M, Pandya DN. 2009. Distinct parietal and temporal pathways to the homologues of Broca's area in the monkey. PLoS Biol. 7(8):e1000170.

Pisella L, Binkofski F, Lasek K, Toni I, Rossetti Y. 2006. No doubledissociation between optic ataxia and visual agnosia: multiple substreams for multiple visuo-manual integrations. Neuropsychologia. 44:2734-2748.

Price CJ. 2000. The anatomy of language: contributions from functional neuroimaging. J Anat. 197:335-359.

Raine A, Yang Y. 2006. Neural foundations to moral reasoning and antisocial behaviour. Soc Cogn Affect Neurosci. 1:203-213.

Rakic P, Goldman-Rakic PS, Gallager D. 1988. Quantitative autoradiography of major neurotransmitter receptors in the monkey striate and extrastriate cortex. J Neurosci. 8:3670-3690.

Rizzolatti G. 2005. The mirror neuron system and its function in humans. Anat Embryol (Berl). 210:419-421.

Rottschy C, Eickhoff SB, Schleicher A, Mohlberg H, Kujovic M, Zilles K, Amunts K. 2007. Ventral visual cortex in humans: cytoarchitectonic mapping of two extrastriate areas. Hum Brain Mapp. 28:1045-1059.

Rozzi S, Calzavara R, Belmalih A, Borra E, Gregoriou GG, Matelli M, Luppino G. 2006. Cortical connections of the inferior parietal convexity of the macaque monkey. Cereb Cortex. 16(10):1389-1417.

Rozzi S, Ferrari PF, Bonini L, Rizzolatti G, Fogassi L. 2008. Functional organization of inferior parietal lobule convexity in the macaque monkey: electrophysiological characterization of motor, sensory and mirror responses and their correlation with cytoarchitectonic areas. Eur J Neurosci. 28(8):1569-1588.

Rushworth MF, Behrens TE, Johansen-Berg H. 2006. Connection patterns distinguish 3 regions of human parietal cortex. Cereb Cortex. 16:1418-1430.

Rushworth MF, Paus T, Sipila PK. 2001. Attention systems and the organization of the human parietal cortex. J Neurosci. 21:5262-5271.

Scheperjans F, Eickhoff SB, Hömke L, Mohlberg H, Hermann K, Amunts K, Zilles K. 2008. Probabilistic maps, morphometry, and variability of cytoarchitectonic areas in the human superior parietal cortex. Cereb Cortex. 18(9):2141-2157.

Scheperjans F, Grefkes C, Palomero-Gallagher N, Schleicher A, Zilles K. 2005. Subdivisions of human parietal area 5 revealed by quantitative receptor autoradiography: a parietal region between motor, somatosensory, and cingulated cortical areas. Neuroimage. 25:975-992.

Scheperjans F, Hermann K, Eickhoff SB, Amunts K, Schleicher A, Zilles K. 2008. Observer-independent cytoarchitectonic mapping of the human superior parietal cortex. Cereb Cortex. 18(4):846-867.

Scheperjans F, Palomero-Gallagher N, Grefkes C, Schleicher A, Zilles K. 2005. Transmitter receptors reveal segregation of cortical areas in 
the human superior parietal cortex: relations to visual and somatosensory regions. Neuroimage. 28:362-379.

Schleicher A, Morosan P, Amunts K, Zilles K. 2009. Quantitative architectural analysis: a new approach to cortical mapping. J Autism Dev Disord. 39(11):1568-1581.

Schleicher A, Palomero-Gallagher N, Morosan P, Eickhoff SB, Kowalski T, Amunts K, Zilles K. 2005. Quantitative architectural analysis: a new approach to cortical mapping. Anat Embryol (Berl). 210(5):373-386.

Seltzer B, Pandya DN. 1984. Further observations on parieto-temporal connections in the rhesus monkey. Exp Brain Res. 55:301-312.

Shalom DB, Poeppel D. 2008. Functional anatomic models of language: assembling the pieces. Neuroscientist. 14(1):119-127.

Tomassini V, Jbabdi S, Klein JC, Behrens TE, Pozzilli C, Matthews PM, Rushworth MFS, Johansen-Berg H. 2007. Diffusion-weighted imaging tractography-based parcellation of the human lateral premotor cortex identifies dorsal and ventral subregions with anatomical and functional specializations. J Neurosci. 27:10259-10269.

Ungerleider LG, Haxby JV. 1994. 'What' and 'where' in the human brain. Curr Opin Neurobiol. 4(2):157-165.

Ungerleider LG, Mishkin M. 1982. Two cortical visual systems. In: Ingle DJ, Goodale MA, Mansfield RJW, editors. Analysis of visual behaviour. Cambridge (MA): MIT Press. p. 549-586.

Van Overwalle F, Baetens K. 2009. Understanding other's actions and goals by mirror and mentalizing systems: a meta-analysis. Neuroimage. 48(3):564-584.
Vigneau M, Beaucousin V, Hervé PY, Duffau H, Crivello F, Houdé O, Mazoyer B, Tzourio-Mazoyer N. 2006. Meta-analyzing left hemisphere language areas: phonology, semantics, and sentence processing. Neuroimage. 30:1414-1432.

von Economo K, Koskinas G. 1925. Die Cytoarchitektonik der Hirnrinde des erwachsenen Menschen. Wien (Austria): Springer.

Vossel S, Thiel CM, Fink GR. 2006. Cue validity modulates the neural correlates of covert endogenous orienting of attention in parietal and frontal cortex. Neuroimage. 32:1257-1264.

Zilles K, Amunts K. 2009. Receptor mapping: architecture of the human cerebral cortex. Curr Opin Neurol. 22(4):331-339.

Zilles K, Palomero-Gallagher N. 2001. Cyto-, myelo-, and receptor architectonics of the human parietal cortex. Neuroimage. 14:S8-S20.

Zilles K, Palomero-Gallagher N, Grefkes C, Scheperjans F, Boy C, Amunts K, Schleicher A. 2002. Architectonics of the human cerebral cortex and transmitter receptor fingerprints: reconciling functional neuroanatomy and neurochemistry. Eur Neuropsychopharmacol. 12:587-599.

Zilles K, Palomero-Gallagher N, Schleicher A. 2004. Transmitter receptors and functional anatomy of the cerebral cortex. J Anat. 205:417-432.

Zilles K, Schleicher A, Palomero-Gallgher N, Amunts K. 2002. Quantitative analysis of cyto- and receptor architecture of the human brain. In: Toga A, Mazziotta J, editors. Brain mapping: the methods. 2nd ed. San Diego (CA): Academic Press. p. 573-602. 